\title{
A Disturbance Rejection Approach to Actuator and Sensor Placement
}

\author{
K.B. $\operatorname{Lim}^{*}$ \\ NASA Langley Research Center \\ Hampton, Virginia 23681-0001
}

\section{Introduction}

For various reasons as discussed for instance in [1], the selection of actuator and sensor positions is still ad hoc. This is especially true for flexible structures where many candidate configurations can exist. This study is an attempt to make the selection process more methodical.

One approach to actuator and sensor placement is to optimize a closed loop performance metric directly by selecting the actuators, sensors, and controller gains simultaneously. This direct approach makes sense if the desired closed loop performance is well defined. Since the individual actuator and sensor contributions to the closed loop performance metric is complex, the solution strategy usually employs non linear programming with many design and numerical iterations. A second approach is to select actuators and/or sensors based on open loop properties so that closed loop performance is indirectly optimized. Since the individual sensor and actuator contributions to the open loop metric is simple, nonlinear optimization is usually not needed. This approach will suggest efficient actuator and sensor configurations for any type of control law. The method suggested in this study falls into the latter class of approaches.

In this note, the use of Hankel singular value (HSV) formula as an actuator placement

\footnotetext{
${ }^{*}$ Research Engineer, Guidance \& Control Branch, Flight Dynamics \& Controls Division, MS 161, k.b.lim@larc.nasa.gov
} 
metric [2] is extended to flexible structures in discrete time [3]. A main novelty introduced is that the ambiguity in the weighting of the principal modes is addressed by incorporating the general disturbance rejection goal into the actuator and sensor placement formulation. Optimal actuator and sensor placement is considered for the purpose of designing control laws for the general disturbance rejection problem. This apparent restriction to disturbance rejection problem is not too restrictive since it is well known from modern multivariable control theory that stability and even robustness requirements can be transformed to this form with appropriate weighting. Simulation results demonstrates that the improvement in closed loop performance is independent of the type of controller used since open loop properties have been improved.

\section{Actuators and Sensors for Disturbance Rejection}

Figure 1 show a schematic of the disturbance rejection problem where the inputs to the plant

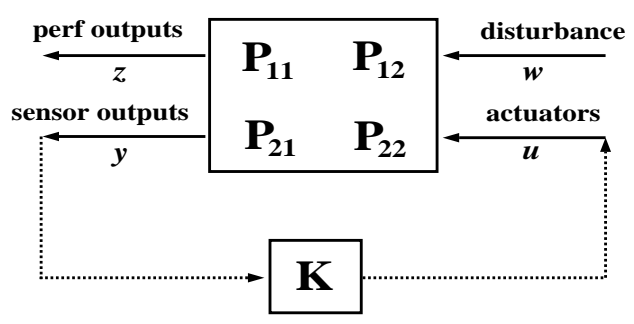

Figure 1: General disturbance rejection problem

consist of two vectors and similarly the outputs consist of two vectors. As a necessary starting point in a disturbance rejection problem, the disturbance source (or inputs) and the output response defining the performance to be optimized is usually defined by the requirements of the control problem.

The disturbance rejection viewpoint taken in this study is similar to the mode selection viewpoint proposed earlier in the context of model reduction for flexible structures [4]. The above formulation is also consistent with the general multivariable control design [5] where the plant $P=\left[P_{11}, P_{12} ; P_{21}, P_{22}\right]$ is assumed given and the problem is to design a stabilizing 
and realizable controller, $K$ to minimize a suitable norm of the closed loop transfer function matrix, $F_{l}(P, K)$. The basic difficulty in the actuator/sensor placement problem is that only $P_{11}$ is given.

In this note, HSV are used to construct a metric that quantifies the degree of controllability and observability for a given set of sensor and actuator configuration. Although the use of HSV to analyze the degree of controllability and observability of a linear system is well established, especially in model reduction applications $[4,6,7]$, the approximate decomposition of the HSV with multiple sensors and actuators in terms of the HSV of all combinations of sensor and actuator pair is new. This result significantly simplifies the design problem of selecting the most effective set of sensors and actuators for flexible structures. The main novelty of the placement strategy introduced in this note is that the HSV from the disturbance to the performance outputs are used to weigh the HSV between candidate actuators to sensor sets. This is possible because in both cases, individual HSV directly corresponds to individual structural modes which is unique to flexible structures.

\section{Decomposition of HSV}

Given the quadruple $\left(A_{z}, B_{z}, C_{z}, D_{z}\right)$ of a discrete LTI state space matrix of a flexible structure, assumed to be lightly damped with distinct eigenvalues, let $(\tilde{A}, \tilde{B}, \tilde{C}, \tilde{D})$ denote the 2 by 2 block diagonal form whose $i$ th block is

$$
\tilde{A}_{i}=\left[\begin{array}{cc}
\operatorname{Re}\left(z_{i}\right) & -\operatorname{Im}\left(z_{i}\right) \\
\operatorname{Im}\left(z_{i}\right) & \operatorname{Re}\left(z_{i}\right)
\end{array}\right] .
$$

Let $\left(z_{i}, v_{i}\right)$ denote the $i$ th eigenvalue and eigenvector pair of $A_{z}$ and $T$ denote the sampling period. The steady-state discrete time controllability grammian, $W_{c_{\infty}}$, and observability grammian, $W_{o_{\infty}}$, satisfies Sylvester equations. The triple $(\tilde{A}, \tilde{B}, \tilde{C})$ is internally balanced

if its grammians are equal and diagonal [7], i.e., $W_{c_{\infty}}=W_{o_{\infty}}=\Gamma^{2}$. The nonnegative real diagonal elements of $\Gamma$ are called the Hankel singular values of the system.

Due to the diagonal dominance property of the discrete controllability and observability 
grammian for flexible structures, the square of the $i$ th HSV is given [3] by

$$
\gamma_{i}^{4} \cong \frac{\operatorname{trace}\left[\tilde{B} \tilde{B}^{T}\right]_{i i} \text { trace }\left[\tilde{C}^{T} \tilde{C}\right]_{i i}}{\left(4 \delta_{i} T\right)^{2}}
$$

where $\delta_{i}=-\frac{1}{T} \operatorname{Re}\left(\ln z_{i}\right)$ and the subscripts $i i$ denotes the $i$ th 2 by 2 block of matrices formed from the inputs and outputs. It is shown [3] that the approximate formula is quite accurate up to frequencies near $90 \%$ of Nyquist frequency.

For $p$ actuators and $q$ sensors, the input and output matrices consist of $p$ columns and $q$ rows, respectively

$$
B_{z}=\left[B_{z_{1}}, \ldots, B_{z_{p}}\right], \quad C_{z}^{T}=\left[C_{z_{1}}^{T}, \ldots, C_{z_{q}}^{T}\right]
$$

Partition the state transformation matrix as follows

$$
V=\left[r_{1}, \ldots, r_{n}\right] ; \quad V^{-T}=\left[l_{1}^{T}, \ldots, l_{n}^{T}\right]
$$

where $r_{i}=\left[\operatorname{Re}\left(v_{i}\right)-\operatorname{Im}\left(v_{i}\right)\right]$, and $l_{i}^{T}$ are $2 n$ by 2 matrices made up of components of $i$ th right and left eigenvectors. The approximate square of the $i$ th HSV in Eq.(2) can be written in terms of individual actuator and sensor

$$
\gamma_{i}^{4} \cong \frac{\sum_{j=1}^{p} f_{i j}^{2} \cdot \sum_{k=1}^{q} g_{i k}^{2}}{\left(4 \delta_{i} T\right)^{2}}=\sum_{j=1}^{p} \sum_{k=1}^{q} \gamma_{i}^{4}(j, k)
$$

where

$$
f_{i k}^{2}=\left\|l_{i} B_{z_{k}}\right\|_{2}^{2} ; \quad g_{i k}^{2}=\left\|C_{z_{k}} r_{i}\right\|_{2}^{2}
$$

$\gamma_{i}^{4}(j, k)$ denotes the squared $i$ th HSV for the $j$ th input and $k$ th output.

The importance of Eq.(5) lies in the decomposition of the HSV of the multivariable flexible structure in terms of the sum of approximate HSV of each actuator and sensor pair. The contribution of each pair appears in a very convenient form from their placement standpoint. The exact HSV are not decomposable in general, i.e.,

$$
\gamma_{i}^{4}=\lambda_{i}\left(\sum_{j=1}^{p} \sum_{k=1}^{q} W_{c j} W_{o k}\right) \quad \neq \sum_{j=1}^{p} \sum_{k=1}^{q} \lambda_{i}\left(W_{c j} W_{o k}\right)
$$




\section{Placement Metric}

Let $\Gamma_{w z}$ denote the HSV from the disturbance to the performance outputs (see Figure 1)

$$
\Gamma_{w z}=\operatorname{diag}\left(\gamma_{w z_{1}}, \ldots, \gamma_{w z_{n}}\right)
$$

Also let $\Gamma_{u y}$ and $\bar{\Gamma}_{u y}$ denote the HSV for $p$ actuators to $q$ sensors and the HSV from a reference set of actuators to a reference set of sensors respectively

$$
\begin{aligned}
& \Gamma_{u y}=\operatorname{diag}\left(\gamma_{u y_{1}}, \ldots, \gamma_{u y_{n}}\right) \\
& \bar{\Gamma}_{u y}=\operatorname{diag}\left(\bar{\gamma}_{u y_{1}}, \ldots, \bar{\gamma}_{u y_{n}}\right)
\end{aligned}
$$

Define the actuator and sensor placement metric as a weighted sum

$$
J \triangleq \sum_{i=1}^{n} \frac{\gamma_{u y_{i}}^{4}}{\bar{\gamma}_{u y_{i}}^{4}} \gamma_{w z_{i}}^{4} \cong \sum_{j=1}^{q} \sum_{k=1}^{p} J_{j k}
$$

where contribution from $j$ th sensor and $k$ th actuator pair is

$$
J_{j k}=\sum_{i=1}^{n}\left(\frac{\gamma_{w z_{i}}^{4}}{\bar{\gamma}_{u y_{i}}^{4}}\right) \gamma_{u y_{i}}^{4}(j, k)
$$

It is necessary to introduce the above weights because the basic idea is to improve the joint controllability and observability for multiple modes and not all modes are equally important physically. HSV can vary by orders of magnitude and therefore a few physically irrelevent modes could dominate an unweighted scalar metric. Hence, the normalizing weight, $\bar{\gamma}_{u y_{i}}^{4}$, is introduced to make each HSV equally important assuming no a priori physical knowledge of individual modes. An example of a normalizing weight is when all actuators and sensors are included. After the normalization, the relative importance of each mode in the control problem can be incorporated by using the weight, $\gamma_{w z_{i}}^{4}$. Modes that are important in the disturbance rejection performance will give larger weights. Note that $J_{j k}$ is basically a weighted trace of $\Gamma_{u y}(j, k)$ squared.

For actuator (sensor) placement only, the set of $q$ sensors ( $p$ actuators) are assumed fixed. The above metric simplifies to

$$
J \cong \sum_{k=1}^{p} J_{k}^{a c t} \quad\left(\sum_{j=1}^{q} J_{j}^{s e n}\right)
$$


where the contribution of the $k$ th actuator ( $j$ th sensor) is

$$
J_{k}^{a c t}=\sum_{i=1}^{n} f_{i k}^{2} w_{i}^{2} \quad\left(J_{j}^{s e n}=\sum_{i=1}^{n} g_{i j}^{2} w_{i}^{2}\right)
$$

The weighting factor for the $i$ th mode is

$$
w_{i}^{2}=\frac{1}{\left(4 \delta_{i} T\right)^{2}} \frac{\gamma_{w z_{i}}^{4}}{\bar{\gamma}_{u y_{i}}^{4}} \sum_{j=1}^{q} g_{i j}^{2} \quad\left(\frac{1}{\left(4 \delta_{i} T\right)^{2}} \frac{\gamma_{w z_{i}}^{4}}{\bar{\gamma}_{u y_{i}}^{4}} \sum_{k=1}^{p} f_{i k}^{2}\right)
$$

Note that $f_{i k}\left(g_{i j}\right)$ denotes the influence of the $k$ th actuator ( $j$ th sensor) on the $i$ th mode. Additional factors that contribute to the above weighting factor include damping, significance with respect to disturbance rejection, and the reference HSV, for the $i$ th mode.

\section{$5 \quad$ Placement Strategy}

Table 1 show the individual contributions for all candidate sensors and actuators. The sum

\begin{tabular}{|c|ccccc|c|}
\hline & \multicolumn{5}{|c|}{ Act \# } & $\begin{array}{c}\text { Sensor } \\
\text { Placement }\end{array}$ \\
\hline & $J_{11}$ & $\cdots$ & $J_{1 k}$ & $\cdots$ & $J_{1 p}$ & $J_{1}^{\text {sen }}$ \\
Sensor \# & $\vdots$ & & $\vdots$ & & $\vdots$ & $\vdots$ \\
& $J_{j 1}$ & $\cdots$ & $J_{j k}$ & $\cdots$ & $J_{j p}$ & $J_{j}^{\text {sen }}$ \\
& $\vdots$ & & $\vdots$ & & $\vdots$ & $\vdots$ \\
& $J_{q 1}$ & $\cdots$ & $J_{q k}$ & $\cdots$ & $J_{q p}$ & $J_{q}^{\text {sen }}$ \\
\hline Actuator & $J_{1}^{a c t}$ & $\cdots$ & $J_{k}^{a c t}$ & $\cdots$ & $J_{p}^{a c t}$ & $J=\sum_{j=1}^{q} \sum_{k=1}^{p} J_{j k}$ \\
\hline Placement
\end{tabular}

Table 1: Actuator and sensor selection table

of each row is given by the last column while the sum of each column is given by the last row. The total metric for all sensors and actuators are given by the sum of the last row or column.

The costs $J_{k}^{a c t}\left(J_{j}^{\text {sen }}\right)$ represents the contribution of the $k$ th actuator ( $j$ th sensor) using all given sensors (actuators). The goal is to maximize the metric $J$ using the least number of actuators and/or sensors. Note that the advantage of the metric applied to flexible structure is that the contribution of each actuator and sensor appears linearly and independently.

For simultaneous actuator and sensor selection problem, we need to maximize the linear sum of non-negative numbers

$$
J=\sum_{j=1}^{q} \sum_{k=1}^{p} J_{j k}
$$


Clearly, a table of nonnegative numbers need to be examined such that $J$ can be approached using the fewest number of actuators and sensors. For actuator (or sensor) only selection problem, a bar chart of

$$
J=\sum_{k=1}^{p} J_{k}^{a c t}=\sum_{j=1}^{q} J_{j}^{s e n}
$$

will indicate the best selection. Actuators (sensors) with small values of $J_{k}^{a c t}\left(J_{j}^{\text {sen }}\right)$ can be removed as the least significant ones.

\section{Concluding Remarks}

The metric and methodology introduced in this paper appears promising in its potential development as a generic tool for actuator and sensor selection for feedback control design of flexible structures. This optimism is based on the advantages evident in using the approximate HSV formula for actuator/sensor selection: HSV of individual pairs can be summed; and it is analytically and computationally simple and avoids direct numerical optimization; and perhaps most importantly, it gives physical insight.

Preliminary simulation results based on $H_{2}$ and $H_{\infty}$ control law designs indicate that closed loop performance can be improved significantly and the performance improvement is independent of the particular type of control law. This consistent improvement is clearly the result of judiciously improved controllability and observability in the open loop system. However, the particular form specified for the disturbance rejection performance requirements strongly affect optimal actuator selection.

\section{References}

[1] Lim, K.B., and Gawronski, W., "Actuator and sensor placement for control of flexible structures," a chapter in: Control and Dynamics Systems, ed. C.T. Leondes, vol.57, Academic Press, San Diego, 1993, pp.109-152. 
[2] Gawronski, W., and Lim, K.B., "Balanced actuator and sensor placement for flexible structures," AIAA paper No. 95-3259, to appear in International Journal of Control.

[3] Lim, K.B., and Gawronski, W., "Analytical formula of Hankel singular values of flexible structures in discrete time," AIAA Guidance, Navigation, and Control Conference, July 29-31, 1996, San Diego, CA, Paper \# 96-3757.

[4] Gregory, Jr., C.Z., "Reduction of large flexible spacecraft models using internal balancing theory," Journal of Guidance, Control and Dynamics, vol.7, 1984, pp.725732.

[5] Boyd, S.P., and Barratt, C.H., Linear Controller Design: Limits of Performance, Prentice Hall, Englewood Cliffs, NJ, 1991, Chapter 2.

[6] Gawronski, W., and Williams, T., "Model reduction for flexible space structures," Journal of Guidance, Control, and Dynamics, vol.14, No.1, 1991, pp.68-76.

[7] Moore, B.C., "Principal component analysis in linear systems: Controllability, observability and model reduction," IEEE Trans. Autom. Control, vol.26, 1981, pp.17-32. 